\title{
Analysis of the Dynamic Response of Multielement Semiconductor Lasers
}

\author{
ROBERT J. LANG, STUDENT MEMBER, IEEE, AND AMNON YARIV, FELlOW, IEEE
}

\begin{abstract}
We present a derivation of the dynamic response of a semiconductor laser consisting of more than one active element. We show that the amplitude and phase of the modulated cavity adiabatically follows the complex resonance of the composite cavity; and using this relation, plus linearized carrier equations, we calculate the parameters characterizing the modulation response of the composite system. In the process, "eff ective" differential gain constants and linewidth enhancement factors arise which take the place of the corresponding parameters in single-element lasers. In the case of a two-section laser, we show that frequency chirping under modulation is present except under special conditions; we identify those conditions and show how chirping can be avoided.
\end{abstract}

\section{INTRODUCTION}

$\mathrm{S}$ INGLE-mode semiconductor lasers (SL's) are desirable for use in fiber optic links because their narrow frequency spread translates into a minimum of pulse spreading in a dispersive fiber. However, most simple Fabry-Perot SL's oscillate in a number of longitudinal modes, particularly under high-frequency modulation. To provide suppression of all but a single mode, more complicated resonator structures have been proposed and demonstrated, including distributed feedback lasers [1], distributed Bragg reflectors [2], axially coupled cavity lasers [3], [4], and laterally coupled cavity lasers [5].

The analysis of such structures is correspondingly more difficult than that of a simple Fabry-Perot cavity. The steady-state lasing frequency can be analyzed by performing a roundtrip analysis of each element of the system including intercavity coupling [5]-[12]. The result of such analyses is typically a nonlinear equation which implicitly defines the lasing frequency $\omega$; the equation depends upon the gain and refractive indexes (and hence, carrier density) of the various cavities, and the requirement that $\omega$ be real (for steady-state operation) imposes a constraint on the set of carrier densities. The full time evolution under large-signal modulation must include the (also nonlinear) carrier rate equations for the different active elements in the system. In one approach, the electric field in each section of the laser is taken as a dynamical variable, and rate equations for both the electric field and carrier densities are solved for a transient excitation [6], [9], [12]. In the

Manuscript received March 18, 1985; revised May 6, 1985. This work was supported by grants from the ITT Corporation, Rockwell, and the National Science Foundation. The work of R. Lang was supported by a fellowship from the National Science Foundation.

The authors are with the Department of Applied Physics, California Institute of Technology, Pasadena, CA 91125. other approach, a single field equation is used which describes a mode of the composite resonator [7]. The latter treatment has the advantage of including fewer dynamical variables. In either case, the rate equations are nonlinear, due to the nonlinear dependence of characteristic parameters (e.g., lasing frequency, photon lifetime, carrier lifetime) on the dynamical variables. Generally, the only way to solve them is numerically.

Recently, Agrawal [12] presented numerical solutions from a small-signal analysis of a two-active-element laser in which the photon density and carrier densities of each cavity and the relative phase between the two cavities were taken as the dynamical variables. The small-signal modulation response, however, can be solved analytically using the modes of the composite resonator. Not only does this give analytic expressions for the small-signal modulation (useful in their own right), but it enables us to see immediately what physical quantities are important and how they affect such things as the relaxation resonance or linewidth enhancement factor. Furthermore, while a smooth, chirpless small-signal response does not guarantee the same large-signal response, a poor small-signal response pretty much rules out the possibility of a reasonable large-signal response. Finally, pathologies in the smallsignal response (e.g., singularities, negative differential gain) can indicate the presence of other properties of interest (e.g., bistability).

In this paper, we derive for the first time an analytic solution to the dynamic response for a general multielement semiconductor laser. We show that the dynamic behavior of the optical field can be obtained from an analytic continuation of the steady-state eigenvalue equation to complex frequency, and that the relevant parameters which characterize the modulation can be written in terms of partial derivatives of the steady-state equation. This equation, plus linearized carrier equations, yield complete expressions for the amplitude and frequency fluctuations under current modulation. We apply the formalism to the case of a two-element semiconductor laser and explicitly calculate the modulation response as a function of frequency in terms of the effective gain, $\alpha$ parameter, and relaxation resonance frequency. We show that frequency modulation (chirping) is generally present in the two-section laser (and, in fact, in any laser of more than two sections); however, it can be eliminated if the operating point is chosen such that the effective $\alpha$ parameters of the two 
cavities are equal [13] or by appropriate splitting of the modulation current [14].

\section{Optical Field}

The connection between the time-varying medium (the modulated laser cavity) and the time evolution of the laser field is not immediately obvious, so we begin by deriving it. Maxwell's equations for a charge-free time-varying medium lead to a wave equation of the form

$$
\nabla^{2} E(x, t)-\frac{1}{c^{2}} \frac{\partial^{2}}{\partial t^{2}}\left[\mu^{2}(x, \hat{t}) E(x, t)\right]=0
$$

where $E(x, \hat{t})$ is the electric field and $\mu(x, \hat{t})$ is the index of refraction varying on a time scale $\hat{t}=\epsilon t, \epsilon<<1$, i.e., much more slowly than optical frequencies. The presence of gain and loss can be accommodated by allowing complex $\mu$ if the loss is approximately constant as a function of frequency. We shall look for solutions of the form

$$
E(x, t)=A(x, \hat{t}) e^{j \psi(t)}
$$

where $A(x, \hat{t})$ is a normalized, also slowly varying field distribution, and $\psi(t)$ is complex to accommodate both amplitude and frequency fluctuations. [The $\hat{t}$ dependence of $A(x, \hat{t})$ is included because the amplitude at distinct points may vary; the average power fluctuation is absorbed into the imaginary part of $\psi(t)$.] Substitute (2) into (1), and the result, to lowest order in $\epsilon$, is

$$
\nabla^{2} A(x, \hat{t})+\frac{\mu^{2}(x, \hat{t})}{c^{2}}\left[\dot{\psi}^{2}-j \ddot{\psi}\right] A(x, \hat{t})=0
$$

where a dot indicates time differentiation. Now we can separate the equation into time-dependent and time-independent parts:

$$
\begin{aligned}
\nabla^{2} A(x, \hat{t})+\frac{\mu^{2}(x, \hat{t})}{c^{2}} \omega^{2}(\hat{t}) A(x, \hat{t}) & =0 \\
\dot{\psi}^{2}-j \ddot{\psi} & \equiv \omega^{2}(\hat{t}) .
\end{aligned}
$$

In (4), time appears only as a parameter. In fact, (4) is precisely the time-independent Helmholtz equation one derives for a time-invariant medium. Consequently, any calculation of the spatial modes [i.e., $A(x, \hat{t})$ ] based on this equation is valid to the same order as this equationin particular, the steady-state equation for a lasing eigenfrequency is valid as an equation for the instantaneous frequency $\omega(\hat{t})$. Equation (5) then gives the time evolution of the optical field as a function of the instantaneous eigenfrequency. We can expand (5) in a series in $\epsilon$ as well, eliminating the nonlinearity and reducing the equation to the simple (and intuitive)

$$
\dot{\psi}=\omega(\hat{t}) \text {. }
$$

We are now done with the perturbation expansions in $\epsilon$, and so for the remainder of the analysis, we will not make a distinction between $t$ and $\hat{t}$.

Of course, a solution for $\psi$ requires an expression for the steady-state $\omega$, and as mentioned previously, for mul- tielement SL's, $\omega$ is generally defined implicitly in a nonlinear equation of the form.

$$
F\left(\omega, n_{1}, \cdots n_{N}\right)=0
$$

for an $N$-active-element laser with carrier densities $\left\{n_{i}\right\}$. (Note that the number of "elements" in this analysis refers to the number of active elements. The presence of passive sections of the laser complicates the resonance function $F$, but does not increase the number of dynamical variables. Thus, an external-cavity laser is a one-activeelement laser; $\mathrm{a} \mathrm{C}^{3}$ laser is a two-active-element laser.) It is not the point of this paper to derive the steady-state equation; that has been done in numerous works for various structures, including two-element axially coupled lasers [7]-[12], multielement axially coupled lasers [6], and two-element laterally coupled lasers [5]. We note, however, that such an equation, although solved for a fixed, real $\omega$, is formally equivalent to a solution of (4) for the instantaneous frequency $\omega(\hat{t})$; consequently, we need not return to first principles to find the dynamic response of a multielement device. We may simply analytically continue the dc resonance equation to complex $\omega$ and use (7) as an instantaneous definition of $\omega(\hat{t})$ in terms of the independent variables $\left\{n_{i}\right\}$.

We shall also require a set of fill factors, defined by

$$
\Gamma_{i} \equiv \frac{p_{i} \sum_{k} V_{k}}{\sum_{k} p_{k} V_{k}}
$$

where $p_{k}$ is the photon density in the $k$ th cavity and $V_{k}$ is the volume of the $k$ th cavity; the summation includes both active and passive elements. In general, $\Gamma_{i} \neq 1$ (as noted by [6]), and in fact, $\Gamma_{i}$ can (and does) vary under modulation. We note that while the $\Gamma$ 's so defined will depend explicitly upon both $\omega$ and the $\left\{n_{i}\right\}, \omega$ is already defined in terms of the independent $\left\{n_{i}\right\}$ by (7).

We now have all of the machinery to carry out the smallsignal analysis of the optical field. We linearize (6) and (7) about a steady-state operating point

$$
\begin{aligned}
& \dot{\psi} \equiv \omega_{0}+\Delta \omega(t)-j \dot{\rho}(t) \\
& n_{i} \equiv n_{i 0}+\left.\nu_{i}(t)(\Delta \omega-j \dot{\rho}) \frac{\partial F}{\partial \omega}\right|_{\omega\left(0, n_{i 0}\right.}+\left.\sum_{i} \nu_{i} \frac{\partial F}{\partial n_{i}}\right|_{\left.\omega 0, n_{i}\right)}=0
\end{aligned}
$$

Divide by $\partial F / \partial \omega$ and take real and imaginary parts to get

$$
\dot{\rho}=\sum_{i} g_{\text {ieff }}^{\prime} \nu_{i}, \quad \Delta \omega=-\sum_{i} m_{\text {ieff }}^{\prime} \nu_{i}
$$

where

$$
g_{\text {icf }}^{\prime} \equiv \operatorname{Im}\left[\frac{\partial F / \partial n_{i}}{\partial F / \partial \omega}\right], \quad m_{\text {eff }}^{\prime} \equiv \operatorname{Re}\left[\frac{\partial F / \partial n_{i}}{\partial F / \partial \omega}\right] .
$$

We call $g_{\text {ieff }}^{\prime}$ and $m_{\text {ieff }}^{\prime}$ the effective differential gain and index constants, respectively. It is clear from the above relations that they have the correct units; it remains to be 
shown that they, in fact, play the same role as the corresponding parameters in single-cavity lasers.

\section{Carrier Dynamics}

Each section of the laser can be treated as an independent carrier pool, described by a volume-averaged rate equation

$$
\begin{array}{ll}
\frac{1}{\tau_{i}} \equiv \frac{1}{\tau_{s}}+g_{i}^{\prime} \Gamma_{i} p, & \omega_{\text {ieff }}^{2} \equiv 2 g_{i} \Gamma_{i} p g_{\text {ieff }}^{\prime}, \alpha_{i e f f} \equiv m_{\text {ieff }}^{\prime} / g_{\text {ieff }}^{\prime} \\
d_{i} \equiv\left(j \Omega+\frac{1}{\tau_{i}}\right), & c_{i k} \equiv p g_{i} \frac{d \Gamma_{i}}{d n_{k}} .
\end{array}
$$

With these definitions, (14) and (15) can be put into matrix form:

$$
\left(\begin{array}{lllll}
j \Omega & 0 & -g_{\text {leff }}^{\prime} & \cdots & -g_{N \mathrm{eff}}^{\prime} \\
0 & 1 & \alpha_{\text {leff }} g_{\text {leff }}^{\prime} & \cdots & \alpha_{N \text { eff }}^{\prime} g_{\text {Neff }}^{\prime} \\
\frac{\omega_{\text {leff }}^{2}}{g_{\text {leff }}^{\prime}} & 0 & c_{11}+d_{1} & \cdots & c_{1 N} \\
\vdots & \vdots & \vdots & & \vdots \\
\frac{\omega_{\text {Neff }}^{2}}{g_{N \text { eff }}^{\prime}} & 0 & c_{N 1} & \cdots & c_{N N}+d_{N}
\end{array}\right)\left(\begin{array}{ccc}
\tilde{\rho} & 0 \\
\Delta \tilde{\omega} & 0 \\
\tilde{\nu}_{1}= & \tilde{e}_{1} \\
\vdots & & \vdots \\
\tilde{\nu}_{N} & & \tilde{e}_{N}
\end{array}\right) .
$$

$$
\dot{n}_{i}=\frac{J_{i}}{q d}-\frac{n_{i}}{\tau_{s}}-g_{i}\left(n_{i}\right) \Gamma_{i}\left(n_{1}, \cdots, n_{N}\right) p
$$

where $J_{i}$ is the $i$ th pump current density, $q$ is the charge on a single carrier, $d$ is the active layer thickness, $\tau_{s}$ is the spontaneous lifetime, $g_{i}$ is the gain constant, $\Gamma_{i}$ is the fill factor mentioned above, and $p$ is the average power density (so that $p$ is proportional to $|\exp j \psi|^{2}$ ). We linearize this set of equations in the same way we did in (9):

$$
\begin{aligned}
J_{i} & \equiv J_{i 0}+q d \cdot e_{i}(t) \\
\dot{\nu}_{i} & =e_{i}-\left[\frac{1}{\tau_{s}}+g_{i}^{\prime} \Gamma_{i} p\right]-2 g_{i} \Gamma_{i} p \rho-\sum_{k} g_{i} p \frac{d \Gamma_{i}}{d n_{k}} \nu_{k}
\end{aligned}
$$

where $g_{i}^{\prime}$ is the material (as opposed to the effective) differential gain constant, and the total derivatives of $\Gamma_{i}$ are given by

$$
\frac{d \Gamma_{i}}{d n_{k}}=\frac{\partial \Gamma_{i}}{\partial n_{k}}-m_{\mathrm{keff}}^{\prime} \frac{\partial \Gamma_{i}}{\partial \operatorname{Re}(\omega)}-g_{\mathrm{keff}} \frac{\partial \Gamma_{i}}{\partial \operatorname{Im}(\omega)} .
$$

The distinction between $\operatorname{Re}(\omega)$ and $\operatorname{Im}(\omega)$ must be made here because the fill factor is not an analytic function of $\omega$. We have also made use of the relations

$$
\operatorname{Re} \frac{\partial \omega}{\partial n_{k}}=\frac{\partial \operatorname{Re} \omega}{\partial n_{k}}, \quad \operatorname{Im} \frac{\partial \omega}{\partial n_{k}}=\frac{\partial \operatorname{Im} \omega}{\partial n_{k}} .
$$

We now Fourier transform (10) and (13). The operator $\partial / \partial t$ becomes a factor $j \Omega$, and the equations become

$$
\begin{aligned}
j \Omega \tilde{\rho}= & \sum_{i} g_{\text {ieff }}^{\prime} \tilde{\nu}_{i}, \quad \Delta \tilde{\omega}=-\sum_{i} m_{i \text { eff }}^{\prime} \tilde{\nu}_{i} \\
j \Omega \tilde{\nu}_{i}= & \tilde{e}_{i}-\left[1 / \tau_{s}+g_{i}^{\prime} \Gamma_{i} p\right] \tilde{\nu}_{i}-2 g_{i} \Gamma_{i} p \tilde{\rho} \\
& -\sum_{k} g_{i} p \frac{d \Gamma_{i}}{d n_{k}} \tilde{\nu}_{k}
\end{aligned}
$$

where a tilde indicates a transformed variable. It is convenient to make some definitions of parameters:
This matrix system can be solved by Cramer's rule to yield

$$
\tilde{\rho}(\Omega)=\frac{\left|\begin{array}{llll}
0 & -g_{\text {leff }}^{\prime} & \cdots & -g_{N \text { eff }}^{\prime} \\
\tilde{e}_{1} & c_{11}+d_{1} & \cdots & c_{1 N} \\
\vdots & \vdots & & \vdots \\
\tilde{e}_{N} & c_{N 1} & \cdots & c_{N N}+d_{N}
\end{array}\right|}{\left|\begin{array}{lllll}
j \Omega & -g_{\text {leff }}^{\prime} & \cdots & -g_{N \text { eff }}^{\prime} \\
\frac{\omega_{\text {leff }}^{2}}{g_{\text {leff }}^{\prime}} & c_{11}+ & d_{1} & \cdots & c_{1 N} \\
\vdots & \vdots & & & \vdots \\
\frac{\omega_{N \text { eff }}^{2}}{g_{\text {Neff }}^{\prime}} & c_{N 1} & & \cdots & c_{N N}+d_{N}
\end{array}\right|} \quad(18 \mathrm{a})
$$




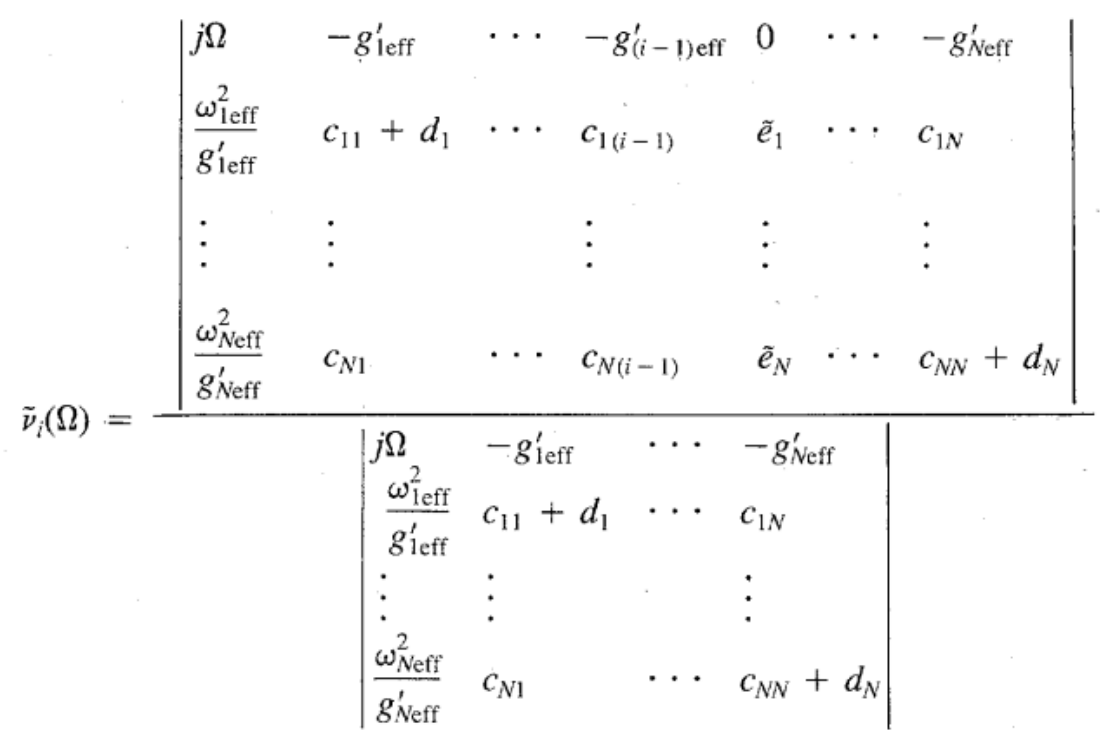

In many cases, the optical power density in an individual element is desired (e.g., the power emitted from one of the exit facets); in this case, the photon density in the $i$ th cavity $p_{i}$ is given by $(8)$ to be

$$
p_{i}=\Gamma_{i} p
$$

which yields the modulation response

$$
\tilde{\rho}_{i}(\Omega)=\Gamma_{i} \tilde{\rho}(\Omega)+\sum_{k} \frac{d \Gamma_{i}}{d n_{k}} \tilde{\nu}_{k}(\Omega)
$$

where the total derivatives $d \Gamma_{i} / d n_{k}$ are defined by (13).

\section{Applications}

We shall first check the formalism by applying it to the well-known case of a single-cavity, simple Fabry-Perot resonator. In this case, $c_{11}$ can be absorbed into the term $1 / \tau_{1}$ (in practice, it can be neglected entirely) and (18) becomes

$$
\tilde{\rho}(\Omega)=\tilde{e}_{1}(\Omega) \frac{\frac{g_{\text {leff }}^{\prime}}{j \Omega+1 / \tau_{1}}}{j \Omega+\frac{\omega_{\text {leff }}^{2}}{j \Omega+1 / \tau_{1}}},
$$$$
\Delta \tilde{\omega}(\Omega)=\tilde{e}_{1}(\Omega) \cdot j \Omega \alpha_{\text {leff }} \frac{\frac{g_{\text {leff }}^{\prime}}{j \Omega+1 / \tau_{1}}}{j \Omega+\frac{\omega_{\text {leff }}^{2}}{j \Omega+1 / \tau_{1}}} .
$$

Equation (21) is exactly the frequency response one derives from the more familiar theory [15], exhibiting a relaxation resonance at frequency $\omega_{\text {leff }}$ and residual phase modulation that goes to zero at zero frequency. Furthermore, we can check the values of $g_{\text {leff }}^{\prime}, \omega_{\text {leff }}$, and $\alpha_{\text {leff }}$ by direct calculation from the simple eigenvalue equation for the lasing frequency:

$$
\begin{aligned}
F\left(\omega, n_{1}\right) \equiv & \frac{1}{R^{2}} \exp \left[\left(\gamma\left(n_{1}\right)-\gamma_{0}\right) L\right. \\
& \left.-\frac{2 j \omega \mu\left(n_{1}\right) L}{c}\right]-1=0
\end{aligned}
$$

where $\gamma\left(n_{1}\right)$ is the power gain per unit length, $\gamma_{0}$ is the loss, $L$ is the length of the laser, $\mu\left(n_{1}\right)$ is the index of refraction, and $R$ is the mirror reflectivity. Applying (10) to $(22)$, we find

which we recognize as being precisely the material differential gain constant, differential index, and linewidth enhancement factor. Similarly, $\omega_{\text {leff }}^{2}$ is the relaxation resonance familiar from McCumber's theory [16].

Thus reassured, we now turn to uncharted territory and analyze the response of a two-element laser. We shall not calculate here the effective modulation quantities; the algebra is straightforward given $F\left(\omega, n_{1}, n_{2}\right)$, but the exact form of $F$ depends on the particular configuration chosen. As above, the diagonal elements $c_{i i}$ can be absorbed into the term $1 / \tau_{i}$ of (16), i.e.,

$$
\frac{1}{\tau_{i}} \equiv \frac{1}{\tau_{s}}+g_{i}^{\prime} \Gamma_{i} p+g_{i} p \frac{d \Gamma_{i}}{d n_{i}} .
$$

$$
\begin{array}{r}
\tilde{\rho}(\Omega)=\tilde{e}_{1}(\Omega)\left\{\frac{\frac{g_{\text {leff }}^{\prime}-g_{\text {eff }}^{\prime} \frac{c_{21}}{j \Omega+1 / \tau_{1}}}{j \Omega+1 / \tau_{2}}}{j \Omega+\frac{\omega_{\text {leff }}^{2}}{j \Omega+1 / \tau_{1}}+\frac{\omega_{2 \mathrm{eff}}^{2}}{j \Omega+1 / \tau_{2}}}\right\} \\
+\tilde{e}_{2}(\Omega)\left\{\frac{\frac{g_{\text {2eff }}^{\prime}-g_{\text {leff }}^{\prime} \frac{c_{12}}{j \Omega+1 / \tau_{2}}}{j \Omega+1 / \tau_{1}}}{j \Omega+\frac{\omega_{\text {leff }}^{2}}{j \Omega+1 / \tau_{1}}+\frac{\omega_{2 \mathrm{eff}}^{2}}{j \Omega+1 / \tau_{2}}}\right\}
\end{array}
$$

$$
g_{\text {leff }}^{\prime}=\frac{\gamma_{c}^{\prime}}{2 \mu}, \quad m_{\text {leff }}^{\prime}=\frac{\omega \mu^{\prime}}{\mu}, \quad \alpha_{\text {leff }}=\frac{2 \omega \mu^{\prime}}{\gamma_{c}^{\prime}}
$$

Then, evaluating (18) for a two-active element laser yields 


$$
\begin{aligned}
& \Delta \tilde{\omega}(\Omega)=\tilde{e}_{1}(\Omega)\left\{\frac{\frac{g_{\text {leff }}^{\prime}}{j \Omega+1 / \tau_{1}}\left[j \Omega\left(\alpha_{\text {leff }}-\alpha_{2 \text { eff }} \frac{g_{\text {2eff }}^{\prime}}{g_{\text {leff }}^{\prime}} \frac{c_{21}}{j \Omega+1 / \tau_{2}}\right)+\frac{\left(\alpha_{\text {leff }}-\alpha_{2 \text { eff }}\right) \omega_{2 \mathrm{eff}}^{2}}{j \Omega+1 / \tau_{2}}\right]}{j \Omega+\frac{\omega_{\text {leff }}^{2}}{j \Omega+1 / \tau_{1}}+\frac{\omega_{2 \mathrm{eff}}^{2}}{j \Omega+1 / \tau_{2}}}\right\} \\
& +\tilde{e}_{2}(\Omega)\left\{\frac{\frac{g_{\text {eff }}^{\prime}}{j \Omega+1 / \tau_{2}}\left[j \Omega\left(\alpha_{2 \mathrm{eff}}-\alpha_{\text {leff }} \frac{g_{\text {leff }}^{\prime}}{g_{\text {2eff }}^{\prime}} \frac{c_{12}}{j \Omega+1 / \tau_{1}}\right)+\frac{\left(\alpha_{2 \mathrm{eff}}-\alpha_{\text {leff }}\right) \omega_{\text {leff }}^{2}}{j \Omega+1 / \tau_{1}}\right]}{j \Omega+\frac{\omega_{\text {leff }}^{2}}{j \Omega+1 / \tau_{1}}+\frac{\omega_{2 \mathrm{eff}}^{2}}{j \Omega+1 / \tau_{2}}}\right\} .
\end{aligned}
$$

The expressions for $\tilde{\nu}_{i}(\Omega)$ are similarly obtainable (and equally formidable); however, the relations simplify somewhat if $c_{i j} \tau_{i} \ll 1$ for all off-diagonal elements $c_{i j}$ (which has been suggested, for example, for the laterally coupled cavity laser [5]). In this case, there is no difference between $\tilde{\rho}(\Omega)$ and $\tilde{\rho}_{i}(\Omega)$, and (24) and (25) reduce to

$$
\begin{aligned}
& \tilde{\rho}(\Omega)=\tilde{e}_{1}(\Omega)\left\{\frac{\frac{g_{\text {leff }}^{\prime}}{j \Omega+1 / \tau_{1}}}{j \Omega+\frac{\omega_{\text {leff }}^{2}}{j \Omega+1 / \tau_{1}}+\frac{\omega_{2 \mathrm{eff}}^{2}}{j \Omega+1 / \tau_{2}}}\right\}+\tilde{e}_{2}(\Omega)\left\{\frac{\frac{\mathrm{g}_{\text {eff }}^{\prime}}{j \Omega+1 / \tau_{2}}}{j \Omega+\frac{\omega_{\text {leff }}^{2}}{j \Omega+1 / \tau_{1}}+\frac{\omega_{2 \mathrm{eff}}^{2}}{j \Omega+1 / \tau_{2}}}\right\} \\
& \Delta \omega(\Omega)=\tilde{e}_{1}(\Omega)\left\{\frac{\frac{g_{\text {leff }}^{\prime}}{j \Omega+1 / \tau_{1}}\left[j \Omega \alpha_{\text {leff }}+\frac{\left(\alpha_{\text {leff }}-\alpha_{\text {2eff }}\right) \omega_{\text {eff }}^{2}}{j \Omega+1 / \tau_{2}}\right]}{j \Omega+\frac{\omega_{\text {leff }}^{2}}{j \Omega+1 / \tau_{1}}+\frac{\omega_{2 \mathrm{eff}}^{2}}{j \Omega+1 / \tau_{2}}}\right\}+\tilde{e}_{2}(\Omega)\left\{\frac{\frac{g_{\text {eff }}^{\prime}}{j \Omega+1 / \tau_{2}}\left[j \Omega \alpha_{2 \mathrm{eff}}+\frac{\left(\alpha_{2 \mathrm{eff}}-\alpha_{\text {leff }}\right) \omega_{\text {leff }}^{2}}{j \Omega+1 / \tau_{1}}\right]}{j \Omega+\frac{\omega_{\text {leff }}^{2}}{j \Omega+1 / \tau_{1}}+\frac{\omega_{2 \mathrm{eff}}^{2}}{j \Omega+1 / \tau_{2}}}\right\} .
\end{aligned}
$$

In Figs. 1 and 2 , we have plotted $\tilde{\rho}(\Omega)$ and $\Delta \tilde{\omega}(\Omega)$ as a function of frequency for a particular set of effective modulation constants with the $c_{i j}$ taken to be zero. The first feature to note is the presence of two resonance terms in the denominators. They combine to give a single resonance at the Pythagorean sum of the two resonance frequencies (as can be seen from Figs. 1 and 2). The resonant frequencies are proportional to $g_{\text {ieff }}^{\prime}$; consequently, any increase in $g_{\text {iff }}^{\prime}$ over the material $g_{i}^{\prime}$ will result in an enhanced modulation bandwidth. Such behavior has been demonstrated in passive-active coupled cavities [13].

Another feature of interest is the fact that the frequency modulation does not go to zero as $\Omega \rightarrow 0$. In fact, while the first term in the square brackets in (27) is simply the residual FM found in any single-cavity semiconductor laser which disappears at zero frequency, the second term causes the frequency response to level off at low frequencies (in fact, it can be seen from (27) that if the difference in the effective $\alpha$ parameters is of order 1 , the frequency response is flat up to the relaxation resonance). This results in an undesirable FM response under modulation. It should be noted that for multielement lasers, the effective linewidth enhancement factor is generally not equal to the material enhancement factor (the corresponding quantity for the single-element laser) -it depends strongly on the particular operating point selected. So, if an operating point is chosen such that the effective $\alpha$ parameters for the two lasers are exactly equal, the low-frequency FM mod- ulation disappears. Alternatively, it may be nulled out by splitting the current modulation such that

$$
\frac{\tilde{e}_{1}}{g_{1} \Gamma_{1}}=\frac{\tilde{e}_{2}}{g_{2} \Gamma_{2}}
$$




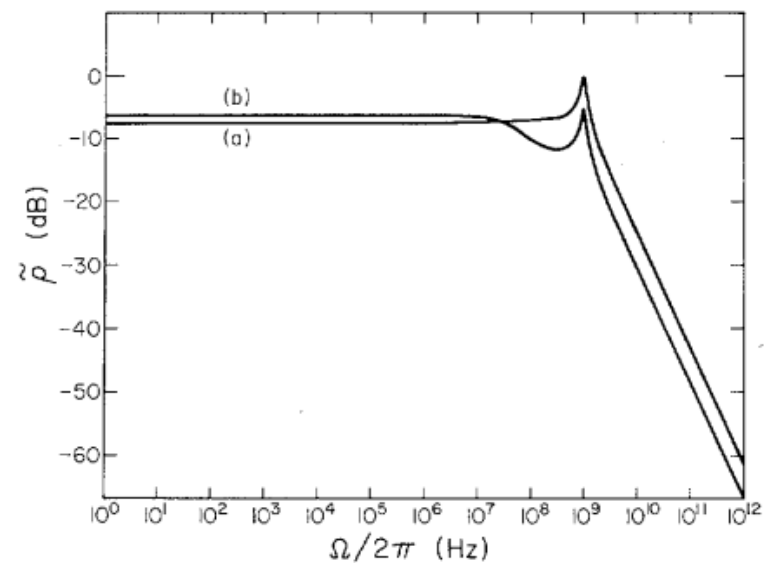

Fig. 1. Amplitude modulation response for parameters $\omega_{\text {ieff }}=6 \cdot \cdot 10^{9} \mathrm{rad} /$ $\mathrm{s}, \omega_{2 \mathrm{eff}}=1 . \cdot 10^{9} \mathrm{rad} / \mathrm{s}, \tau_{1}=10^{-9} \mathrm{~s}, \tau_{2}=5 \cdot 10^{-9} \mathrm{~s}, g_{\text {ieff }}^{\prime}=1.2$. $10^{-5} \mathrm{~cm}^{3} / \mathrm{s}, g_{2 \mathrm{eff}}^{\prime}=3.0 \cdot 10^{-6} \mathrm{~cm}^{3} / \mathrm{s}, \alpha_{1 \mathrm{effi}}=5.01, \alpha_{2 \mathrm{eff}}=5.00$. (a) Amplitude modulation response (arbitrary zero) for $\tilde{e}_{2}=0$. (b) Amplitude modulation response (same zero) for $\tilde{e}_{1}=0$.

\section{REFERENCES}

[1] M. Nakamura, K. Aiki, J-I Umeda, A. Yariv, H. W. Yen, and T. Morkawa, "GaAs-GaAlAs double heterostructure distributed feedback diode lasers," Appl. Phys. Lett., vol. 25, p. 487, 1974.

[2] H. W. Yen, W. Ng, I. Samid, and A. Yariv, "GaAs distributed Bragg reflector lasers," Opt. Commun., vol. 17, p. 213, 1976.

[3] W. T. Tsang and N. A. Olsson, "High speed direct single-frequency modulation with large tuning rate and frequency excursion in cleavedcoupled-cavity semiconductor lasers," Appl. Phys. Lett., vol. 42, p. $650,1983$.

[4] K. J. Ebeling, L. A. Coldren, B. I. Miller, and J. A. Rentschler, "Single-mode operation of coupled-cavity GalnAsP/InP semiconductor lasers," Appl. Phys. Lett., vol. 42, p. 6, 1983.

[5] J. Salzman, R. Lang, and A. Yariv, "The laterally coupled cavity laser," vol. 47, p. 195, 1985.

[6] K. J. Ebeling and L. A. Coldren, "Analysis of multielement semiconductor lasers," J. Appl. Phys., vol. 54, p. 2962, 1983.

[7] D. Marcuse and T.-P. Lee, "Rate equation model of a coupled-cavity laser," IEEE J. Quantum Electron., vol. QE-20, p. 166, 1984.

[8] H. K. Choi, K-L. Chen, and S. Wang, "Analysis of two-section coupled-cavity semiconductor lasers," IEEE J. Quantum Electron., vol. QE-20, p. 385, 1984.

[9] L. Coldren and T. L. Koch, "Analysis and design of coupled-cavity lasers-Part I: Threshold gain analysis and design guidelines," IEEE J. Quantum Electron., vol. QE-20, p. 659, 1984.

[10] W. Streifer, D. Yevick, T. L. Paoli, and R. D. Burnham, "An analysis of cleaved coupled-cavity lasers," IEEE J. Quantum Electron., vol. QE-20, p. 754, 1984.

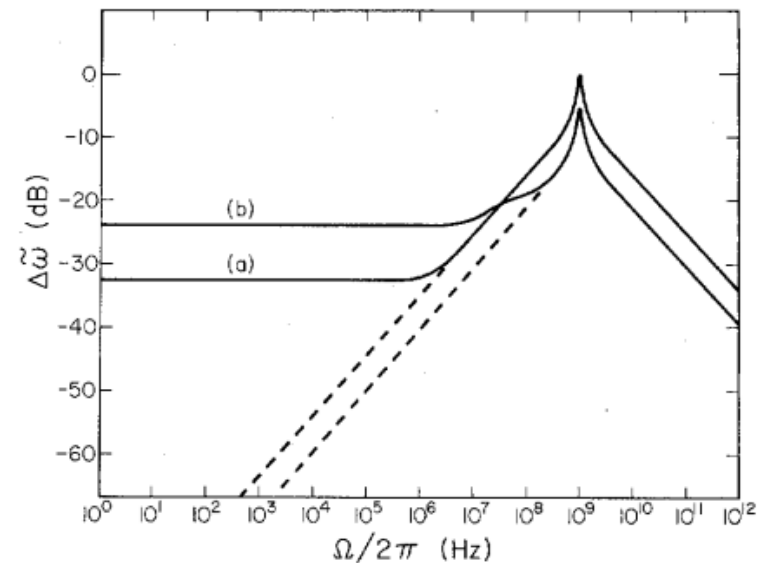

Fig. 2. Frequency modulation response for same parameters as in Fig. 1. (a) $\tilde{e}_{2}=0$, (b) $\hat{e}_{1}=0$. Dashed lines indicate the residual phase modulation present in single-cavity lasers.

[11] C. H. Henry and R. F. Kazarinov, "Stabilization of single-frequency operation of coupled-cavity lasers," IEEE J. Quantum Electron., vol. QE-20, p. 733, 1984.

[12] G. Agrawal, "Coupled-cavity semiconductor lasers under current modulation: Small-signal analysis," IEEE J. Quantum Electron., vol. QE-21, p. 255, 1985.

[13] K. Vahala, J. Paslaski, and A. Yariv, "Observation of modulation speed enhancement, frequency modulation suppression, and phase noise reduction by detuned loading in a coupled-cavity semiconductor laser," Appl. Phys. Lett., vol. 46, p. 1025, 1985.

[14] L. A. Coldren, G. D. Boyd, J. E. Bowers, and C. A. Burrus, "Reduced dynamic linewidth in three-terminal two-section diode lasers," Appl. Phys. Lett., vol. 46, p. 125, 1985.

[15] K. Y. Lau, N. Bar-Chaim, I. Ury, Ch. Harder, and A. Yariv, "Direct amplitude modulation of short-cavity GaAs lasers up to X-band frequencies," Appl. Phys. Lett., vol. 43, p. 1, 1983.

[16] D. E. McCumber, "Intensity fluctuations in the output of CW laser oscillators I," Phys. Rev., vol. 141, p. 306, 1966.

[17] W. Streifer, D. Yevick, T. L. Paoli, and R. D. Burnham, "Cleavedcoupled cavity laser analysis," presented at OSA, San Diego, CA, Oct. 29-Nov. 2, 1984, paper Th04.

Robert J. Lang (S'83), for a photograph and biography, see p. 451 of the May 1985 issue of this JournaL.

Amnon Yariv (S'56-M'59-F'70), for a photograph and biography, see p. 138 of the February 1985 issue of this JournaL. 